\title{
Current and emerging therapies for neuromyelitis optica
}

\author{
Cong Zhao, Hong-Zeng Li, Ya-Nan Bai, Zhu-Yi Li, Jun Guo \\ Department of Neurology, Tangdu Hospital, The Fourth Military Medical University, Xi'an 710038, Shaanxi, China.
}

\section{A B S T RA C T}

Neuromyelitis optica (NMO) is an autoimmune demyelinating disease that mainly affects the optic nerve and spinal cord, potentially resulting in blindness and paralysis. Once thought to be a clinical variant of multiple sclerosis, NMO is currently considered as a different disease with its own features due to the identification of a specific autoantibody against aquaporin 4. Given the high risk of disability, treatment should be launched once the diagnosis is established. Evidence from clinical practice showed that traditional immunosuppressive agents affecting the function of $\mathrm{T}$ and $\mathrm{B}$ cells could attenuate disease exacerbation. Recently, with better understanding pathogenesis of NMO, increasing bodies of novel therapies and therapeutic targets have been discovered. In this review, the authors discuss the current strategies of treating NMO in details and briefly introduce the potential therapies in future.

Key words: Neuromyelitis optica; aquaporin 4; therapies

\section{INTRODUCTION}

Neuromyelitis optica (NMO) is an inflammatory demyelinating disease of the central nervous system (CNS) characterized by recurrent optic neuritis and transverse myelitis. A relapsing-remitting clinical course was observed in the most patients. Historically, NMO was thought to be a variant of multiple sclerosis (MS). Given its distinct clinical features, radiological changes and the identification of the autoantibody targeted to aquaporin 4 (AQP4), ${ }^{[1]}$ it is now believed to be a different disease with its own diagnostic criteria, prognosis and treatment.

The epidemiology information of NMO is limited because of its rarity and commonly confusing with MS. Estimated incidence and prevalence of NMO ranges from 0.05-0.4 and 0.1-4.4 per 100,000 respectively. ${ }^{[2-5]}$ It has a preference in non-Caucasian women. ${ }^{[6]}$ The median age of disease onset is between 34-43 years, while children and elder people are also affected. ${ }^{[7]}$ Unlike MS, patients with NMO are more possibly associated with other autoimmune diseases, like

\section{Corresponding Author: Dr. Jun Guo, Department of \\ Neurology, Tangdu Hospital, The Fourth Military Medical University, Xi'an 710038, Shaanxi, China. \\ Email: guojun_81@163.com}

\begin{tabular}{|c|l|}
\hline \multicolumn{2}{|c|}{ Access this article online } \\
\hline Quick Response Code: & Website: \\
\hline & http://www.nnjournal.net \\
\hline & \\
\hline
\end{tabular}

systemic lupus erythematosus, Sjogren's syndrome, ${ }^{[7]}$ suggesting these patients may have a genetic tendency to autoimmune.

AQP4-IgG, a sero-biomarker of NMO, distinguishes NMO from other demyelinating disorders and was incorporated into the 2006 diagnostic criteria of $\mathrm{NMO}^{\left[{ }^{[8]}\right.}$ broadening the spectrum of NMO. In 2007, neuromyelitis optica spectrum disorder (NMOSD) was termed to encompass patients who have detectable serum AQP4-IgG but do not fully meet the diagnostic criteria (e.g. first-atack LETM, typical NMO with brain lesions, NMO with other autoimmune diseases). ${ }^{[9]}$ In 2015, the international panel for NMO diagnosis unified the term of NMO and NMOSD, and developed diagnostic criteria for seropositive and seronegative NMOSD, respectively. ${ }^{[10]}$

The pathogenesis of NMO still remains ambiguous. It is currently considered that AQP4-IgG is of great importance in triggering NMO. AQP4 is a transmembrane protein expressed by astrocytes and controls the flow of water in cells. ${ }^{[1]}$ Upon the penetration through the blood brain barrier (BBB) and binding with AQP4 on perivascular astrocyte end feet, AQP4-IgG activates the classical complement pathway

This is an open access article distributed under the terms of the Creative Commons Attribution-NonCommercial-ShareAlike 3.0 License, which allows others to remix, tweak, and build upon the work non-commercially, as long as the author is credited and the new creations are licensed under the identical terms.

For reprints contact: service@oaepublish.com

Cite this article as: Zhao $\mathrm{C}, \mathrm{Li} \mathrm{HZ}$, Bai YN, Li ZY, Guo J. Current and emerging therapies for neuromyelitis optica. Neuroimmunol Neuroinflammation 2016;3:127-32.

Received: 03-03-2016; Accepted: 13-04-2016 
which injured astrocytes, followed by marked NK cell, granulocyte and monocyte infiltration along with BBB breakdown, oligodendrocyte death and demyelination, microglia activation and even neuronal apoptosis. ${ }^{[12]}$ Unfortunately, the mechanism of AQP4-IgG generation remains unclear; predictions include AQP4 molecular mimicry and infection. ${ }^{[13,14]}$ Although $\mathrm{T}$ cells are rare in the CNS lesions, recent studies indicate that some $\mathrm{T}$ cell subsets may also involve in the pathological process. Th17 cells ${ }^{[13]}$ and follicular helper $\mathrm{T}$ cells $(\mathrm{Tfh})^{[15]}$ significantly increase during the relapse period, which are critical for breaking down BBB and antibody production. Further investigation into the pathogenesis mechanism of NMO may facilitate the development of more therapeutic targets.

\section{CURRENT THERAPIES OF NMO}

Since NMO has a relapsing-remitting clinical course, the treatment of NMO is divided into two stages: management of acute attacks and prevention of future relapses. The main aims of acute therapies are to suppress the inflammatory response, minimize the irreversible neurological damages and accelerate recovery, whereas preventive therapies are designed to reduce relapse frequency. Treatment strategies for these two stages are discussed as follows.

\section{Therapies of acute attacks}

The most typical treatment for an acute attack of NMO is the administration of intravenous methylprednisolone (IVMP). Glucocorticoids exert profound anti-inflammatory and immunosuppressive effects, including down regulation of pro-inflammatory cytokines, reduction of adhesion molecules and matrix metalloproteinases, prevention of apoptosis of mononuclear cells and restoration of the BBB integrity. ${ }^{[16]} \mathrm{Up}$ to now, there have been no prospective clinical trials for acute treatment of NMO, meaning that the reliable dosages and duration have not been reviewed. Methylprednisolone is commonly prescribed for 1,000 mg per day for 3-5 days, followed by prednisone starting from $60-100 \mathrm{mg}$ per day. If patients demonstrated no improvement after IVMP, plasma exchange (PE) should be considered. PE is recommended as a second-line therapy for refractory patients, accelerating the removal of antibodies, complements, pro-inflammatory cytokines and chemokines. Generally PE is performed daily or every other day for five cycles each of which remove 1.0-1.5x volumes of plasma. ${ }^{[17]}$ Retrospective studies and case series have reported that the total remission rate of PE therapy was between $44-75 \% .{ }^{[18]}$ Male patients and early initiation therapy were associated with better improvement. ${ }^{[18]}$ If patients demonstrated promising improvement after PE, it can be considered as the first-line therapy for the next acute attack. The treatment with intravenous immunoglobulin (IVIg), which is an ideal alternative for PE in other neurological disorders such as myasthenia gravis, multiple sclerosis and Guillain-Barré syndrome, in acute NMO has also been reported; ${ }^{[19,20]}$ however, the improvement of the prognosis of steroid refractory individuals has not been clearly demonstrated, indicating IVIg therapy may need further investigation in acute NMO.

\section{Prevention of acute attacks}

Given its high recurrence rate and severe morbidity associated with one relapse, long-term immunosuppressive treatment should be instituted right after the recovery of the first attack. Due to its low incidence, data of prospective randomized controlled trials of preventive agents are still limited. For developing a treatment protocol, the effects of prior treatment, the short-term and long-term side effects, other system complications and even economic status of patients should be considered intensively.

\section{Azathioprine}

Azathioprine is shown to be the most popular oral immunosuppressive agent currently used in NMO treatment, which can effectively disrupt purine synthesis and inhibit the proliferation of $\mathrm{T}$ and B cells. A large retrospective study including 99 patients with NMO or NMOSD demonstrated that the annualized relapse rate (ARR) decreased from 2.20 to 0.52 during a median treatment interval of 22 months. ${ }^{[21]}$ The usual initial dose is $50 \mathrm{mg} /$ day, and subsequently increased to $2-3 \mathrm{mg} / \mathrm{kg}$ per day, which provides better improvement. As azathioprine may take full effect only after 3-6 months, oral steroids should be used simultaneously ( $1 \mathrm{mg} / \mathrm{kg}$ per day). A recent prospective trial has showed azathioprine plus low dose corticosteroid distinctly reduced the ARR (from 0.923 to 0) of the NMO patients in Southern China and $57.1 \%$ of patients were relapse-free during a median follow-up of 20 months. ${ }^{[22]}$

\section{Mycophenolate mofetil}

Mycophenolate mofetil is a potent immunosuppressive drug that inhibits the inosine monophosphate dehydrogenase and suppresses the proliferation of $\mathrm{T}$ and B cells. It has been demonstrated in a retrospective case series of 24 patients (median dose $2 \mathrm{~g}$ daily) that mycophenolate mofetil decreased the ARR from 1.28 to 0.09 over a median follow-up of 28 months, but the expanded disability scale score (EDSS) remained almost unchanged (6.0 pretreatment vs. 5.5. post-treatment). ${ }^{[23]}$

\section{Mitoxantrone}

Mitoxantrone, an inhibitor of topoisomerase II, is 
firstly used in treatment acute leukemia and other malignancies. The potent ability of suppressing the development of some lymphocytes, especially B cells, guaranteed the therapeutic effect in treatment of some autoimmune diseases, such as aggressive relapsing MS. It was reported in 2006 among 5 patients with NMO. ${ }^{[24]}$ Although 2 patients experienced relapse during a 2-year follow-up period, 4 of them benefited from the treatment $\left(12 \mathrm{mg} / \mathrm{m}^{2}\right.$ monthly for 6 months and 3 cycles at 3-month interval for maintenance) and 3 gained longterm remission. In another case, Kim et al. ${ }^{[25]}$ reported that 20 NMO patients infused with methotrexate (3-6 monthly cycles of $12 \mathrm{mg} / \mathrm{m}^{2}$ followed by $6-12 \mathrm{mg} / \mathrm{m}^{2}$ maintenance doses) showed a reduction of ARR (2.8 to 0.7) and EDSS (5.6 to 4.4).

\section{Methotrexate}

Methotrexate inhibits purine and thymidylate synthesis. A retrospective case of $14 \mathrm{NMO} / \mathrm{NMOSD}$ patients treated with methotrexate at a median dose of $17.5 \mathrm{mg}$ weekly showed a significant decrease of ARR from 1.39 to 0.18 . However, 13 out of 14 patients were co-treated with other immunosuppressive agents: oralprednisolone $(n=11)$, rituximab $(n=1)$, or tacrolimus ( $n=1$ ), and the impact of these remains unknown. ${ }^{[26]}$

\section{Oral corticosteroids}

In addition to intravenous administration of high dose for acute exacerbation, low dose of oral corticosteroids have been shown to be effective in maintaining long-term remission. A cohort of 25 Japanese patients, receiving low-dose prednisolone (2.5-20.0 mg daily) as monotherapy was retrospectively evaluated in a median period of 19.3 months. Results showed that ARR declined from 1.48 to 0.49 . Meanwhile, this study indicated the therapeutic effect was dose-dependent. Patients receiving less than $10 \mathrm{mg} /$ day were more susceptible to relapse. ${ }^{[27]}$

\section{Rituximab}

Rituximab (RTX) is a chimeric mouse/human anti-CD20 monoclonal antibody that specifically depletes peripheral CD20-positive B cells. This antibody binds to the surface antigen on B cells and activates complement-dependent cytotoxicity and antibody-dependent cell-mediated cytotoxicity. It was firstly used in the treatment of lymphomas and leukemia. Recently, RTX has been reported to be effective in treating certain autoimmune disorders such as rheumatoid arthritis. ${ }^{[28]}$ RTX potently suppresses autoimmune reaction by blocking the differentiation of $\mathrm{B}$ cells into plasma cells, antigen expression and release of cytokines simultaneously.
Cree et al. ${ }^{[29]}$ firstly reported that RTX is refractory to other immunosuppressive agents in an open labeled trial of treatment of NMO. In this study, RTX was infused weekly at $375 \mathrm{mg} / \mathrm{m}^{2}$ for 4 weeks followed by $1,000 \mathrm{mg}$ reinfusion if CD19+ B cells were detectable in the peripheral blood. Positive curative effect was observed in all cases with significant reduction of ARR (2.6 to 0) and EDSS (7.5 to 5.5). In another 5-year prospective study, Kim et al. ${ }^{[0]}$ reported that 26 of 30 patients benefited from the treatment with a reduction of ARR and stabilization of EDSS 18 patients totally recovered in 24 months.

Dose-dependent strategies have been exploded in the treatment of NMO. Generally, there are two different regimens: one is discussed above in Cree's study while the other one is $1 \mathrm{~g}$ infusion at an interval of 2 weeks for twice. The dose of RTX was adopted from the treatment of B-cell malignancies which is considerably expensive and has a high probability of occurrence of side effects. A Chinese group reported that repeated infusion with reduced dosage of RTX to five patients with NMO/ NMOSD was very effective in modulation of peripheral B cells and prevention of relapse. ${ }^{[31]}$ The regimen was $100 \mathrm{mg}$ per week for consecutive 3 weeks. One hundred milligram of RTX was reinfused when the percentage of the B cells circulation reached $1 \%$. None of the 5 patients experienced relapse during the 1-year followup period. The median EDSS score slightly decreased from 4.5 to 4 , while all the patients got a significant functional improvement after therapy. Despite the fact that this study indicated that responsiveness may achieve in a lower dose in Asian patients, further investigations with a sufficient number of patients are needed.

\section{OTHERPROMISING THERAPIES}

\section{Complement-targeted therapy}

As discussed above, complement activation pays an important role in the pathogenesis of NMO. Eculizumab, a humanized monoclonal anti-complement C5 antibody, inhibits its cleavage by C5 convertase and subsequently blocks the complement cascade. In an open label clinical trial of 14 patients, intravenously administration of $900 \mathrm{mg}$ eculizumab every 2 weeks significantly reduced ARR and improved the patients' neurological deficits. ${ }^{[32]}$ Twelve of 14 patients got no relapse after 12-month treatment, however one patient got meningococcal sepsis and sterile meningitis. In spite of this preliminary finding, the efficacy and safety of eculizumab should be further reviewed.

C5 is the key molecule of all the three complement pathways. The infectious adverse effect above was likely due to the inhibition of the alternative and lectin 
pathways that are important in eliminating microbes. C1 is only involved in the classical pathway; inhibition C1 not only blocks the cytotoxicity (CDC) and antibody-dependent cellular cytotoxicity (ADCC) effect, but also saves the anti-infection function of the alternative and lectin pathway. It has been discovered that C1-inhibiting monoclonal antibody is sufficient for preventing NMO in mouse models. ${ }^{[33]}$ Another small-scale phase I clinical trial reported C1 esterase inhibitor significantly reduced the EDSS score of patients with NMO, and no obvious side effects showed up. ${ }^{[34]}$ So the selective inhibition of the classical complement pathway is hypothesized as another more promising and safer complementtargeted therapy.

\section{Inhibition of IL-6-IL-6 receptor signaling}

A few studies have indicated that interleukin 6 (IL-6), increasing in NMO during relapse phase, is a potent driver of NMO relapsing to enhance the survival of plasma blast and secretion of AQP4IgG. ${ }^{[15,35-37]}$ Tocilizumab, an IL-6 receptor blocking antibody, reduced the survival of AQP4-IgGsecreting plasma blast and reduced NMO relapse. A few case reports showed that tocilizumabhasa positive impact on the patients' condition due to its ability of suppressing the CD20-plasma cells and decreasing the titer of AQP4-IgG, ${ }^{[38-40]}$ suggesting tocilizumab is a promising alternative therapy for aggressive, immunosuppressive agents-refractory patients. Clinical trials in large scale are needed to examine the effectiveness and safety of tocilizumab in NMO treatment.

\section{Restoring stability of blood brain barrier}

Elevated level of albumin was detected in the CSF of patients with NMO during relapse, indicating the stability of $\mathrm{BBB}$ is damaged in the acute phase of NMO. ${ }^{[41]}$ A few studies reported serum from AQP4-IgG positive patients increases the permeability of artificial $\mathrm{BBB}$ and reduces the expression of tight junction proteins. ${ }^{[42,43]}$ Although the specific mechanism still remains unclear, majority of studies strongly suggested that the vascular endothelial growth factor (VEGF) ${ }^{[42]}$ and matrix metalloproteinases ${ }^{[44]}$ were involved. Currently the anti-VEGF monoclonal antibody, bevacizumab, is under clinical review of the clinical benefits in restoring the stability of $\mathrm{BBB}$ in $\mathrm{NMO}$ patients. ${ }^{[45]}$

\section{Restraining the activity of neutrophils}

Large number of neutrophils was detected in the perivascular lesions in both human cases of ${ }^{[46]}$ and animal model ${ }^{[47]}$ of NMO. Sivelestat, a neutrophil protease inhibitor, has been demonstrated with the inhibitory effect on migration of neutrophils and reductive effect on neutrophil-related tissue damages in a NMO model of mouse. ${ }^{[4]}$ The authors further observed that neutrophils enter the CNS within $24 \mathrm{~h}$ after onset, ${ }^{[47]}$ suggesting that Sivelestat may be effective in the treatment of acute NMO.

Eosinophils infiltration into the demyelinating lesions is another pathological hallmark of NMO. ${ }^{[48]}$ Degranulation of mouse bone marrow-derived eosinophils significantly enhanced the AQP4-IgG-mediated ADCC and CDC effect in cultured spinal cord slices, indicating eosinophils granules are involved in the pathogenic process of NMO. ${ }^{[48]}$ In the same study, cetirizine, the second generation of antihistamine, reduces tissue damage in a NMO model of mouse, which may be another option to control NMO. ${ }^{[48]}$

\section{CONCLUSION}

Corticosteroids, azathioprine, mycophenolate mofetil and rituximab are still the first-line therapy for NMO. With the dramatically increasing knowledge of NMO, a variety of novel therapies are under test and development, including complement-targeted therapy, IL-6 targeted therapy, restoring the stability of BBB and granulocytes inhibition, etc. The ultimate goal of NMO therapeutics is to develop a highly selective, low side effect therapy. This is challenging since clinical experience of treating $\mathrm{NMO}$, a rare disease, is limited. So multiple-center, large-scale randomized controlled trials for treatment-naïve patients are needed to review the efficacy of these therapeutic approaches.

\section{Acknowlegments}

This work was supported by the New Clinical Technique or Program of Tangdu Hospital in 2013 and 2014.

\section{Financial support and sponsorship}

Nil.

\section{Conflicts of interest}

There are no conflicts of interest.

\section{REFERENCES}

1. Lennon VA, Wingerchuk DM, Kryzer TJ, Pittock SJ, Lucchinetti CF, Fujihara K, Nakashima I, Weinshenker BG. A serum autoantibody marker of neuromyelitis optica: distinction from multiple sclerosis. Lancet 2004;364:2106-12.

2. Cabre P, Heinzlef O, Merle H, Buisson GG, Bera O, Bellance R, Vernant JC, Smadja D. MS and neuromyelitis optica in Martinique (French West Indies). Neurology 2001; 56:507-14.

3. Cabrera-Gomez JA, Kurtzke JF, Gonzalez-Quevedo A, LaraRodriguez R. An epidemiological study of neuromyelitis optica in Cuba. J Neurol 2009;256:35-44.

4. Asgari N, Lillevang ST, Skejoe HP, Falah M, Stenager E, Kyvik KO. A population-based study of neuromyelitis optica in Caucasians. Neurol 2011;76:1589-95. 
5. Pandit L, Asgari N, Apiwattanakul M, Palace J, Paul F, Leite MI, Kleiter I, Chitnis T; GJCF International Clinical Consortium \& Biorepository for Neuromyelitis Optica. Demographic and clinical features of neuromyelitis optica: a review. Mult Scler 2015;21:845-53.

6. Flanagan EP, Cabre P, Weinshenker BG, St Sauver J, Jacobson DJ, Majed M, Lennon VA, Lucchinetti CF, McKeon A, Matiello M, Kale N, Wingerchuk DM, Mandrekar J, Sagen JA, Fryer JP, Borders Robinson A, Pittock SJ. Epidemiology of aquaporin-4 autoimmunity and neuromyelitis optica spectrum. Ann Neurol 2016; doi: 10.1002/ ana.24617.

7. Jarius S, Ruprecht K, Wildemann B, Kuempfel T, Ringelstein M, Geis C, Kleiter I, Kleinschnitz C, Berthele A, Brettschneider J, Hellwig K, Hemmer B, Linker RA, Lauda F, Mayer CA, Tumani H, Melms A, Trebst C, Stangel M, Marziniak M, Hoffmann F, Schippling S, Faiss JH, Neuhaus O, Ettrich B, Zentner C, Guthke K, Hofstadt-van Oy U, Reuss R, Pellkofer H, Ziemann U, Kern P, Wandinger KP, Bergh FT, Boettcher T, Langel S, Liebetrau M, Rommer PS, Niehaus S, Munch C, Winkelmann A, Zettl UU, Metz I, Veauthier C, Sieb JP, Wilke C, Hartung HP, Aktas O, Paul F. Contrasting disease patterns in seropositive and seronegative neuromyelitis optica: a multicentre study of 175 patients. $J$ Neuroinflammation 2012;9:14.

8. Wingerchuk DM, Lennon VA, Pittock SJ, Lucchinetti CF, Weinshenker BG. Revised diagnostic criteria for neuromyelitis optica. Neurology 2006;66:1485-9.

9. Wingerchuk DM, Lennon VA, Lucchinetti CF, Pittock SJ, Weinshenker BG. The spectrum of neuromyelitis optica. Lancet Neurol 2007;6:805-15.

10. Wingerchuk DM, Banwell B, Bennett JL, Cabre P, Carroll W, Chitnis T, de Seze J, Fujihara K, Greenberg B, Jacob A, Jarius S, Lana-Peixoto M, Levy M, Simon JH, Tenembaum S, Traboulsee AL, Waters P, Wellik KE, Weinshenker BG; International Panel for NMO Diagnosis. International consensus diagnostic criteria for neuromyelitis optica spectrum disorders. Neurology 2015;85:177-89.

11. Agre P, Kozono D. Aquaporin water channels: molecular mechanisms for human diseases. FEBS Lett 2003;555:72-8.

12. Papadopoulos MC, Verkman AS. Aquaporin 4 and neuromyelitis optica. Lancet Neurol 2012;11:535-44.

13. Varrin-Doyer M, Spencer CM, Schulze-Topphoff U, Nelson PA, Stroud RM, Cree BA, Zamvil SS. Aquaporin 4-specific T cells in neuromyelitis optica exhibit a Th17 bias and recognize Clostridium ABC transporter. Ann Neurol 2012;72:53-64.

14. Koga M, Takahashi T, Kawai M, Fujihara K, Kanda T. A serological analysis of viral and bacterial infections associated with neuromyelitis optica. J Neurol Sci 2011;300:19-22

15. Li YJ, Zhang F, Qi Y, Chang GQ, Fu Y, Su L, Shen Y, Sun N, Borazanci A, Yang C, Shi FD, Yan Y. Association of circulating follicular helper T cells with disease course of NMO spectrum disorders. $J$ Neuroimmunol 2015;278:239-46.

16. Barnes PJ. Corticosteroids: the drugs to beat. Eur $J$ Pharmacol 2006;533:2-14.

17. Bienia B, Balabanov R. Immunotherapy of neuromyelitis optica. Autoimmune Dis 2013;2013:741490.

18. Bonnan M, Cabre P. Plasma exchange in severe attacks of neuromyelitis optica. Mult Scler Int 2012;2012:787630.

19. Elsone L, Panicker J, Mutch K, Boggild M, Appleton R, Jacob A. Role of intravenous immunoglobulin in the treatment of acute relapses of neuromyelitis optica: experience in 10 patients. Mult Scler 2014;20:501-4.

20. Okada K, Tsuji S, Tanaka K. Intermittent intravenous immunoglobulin successfully prevents relapses of neuromyelitis optica. Intern Med 2007;46:1671-2.

21. Costanzi C, Matiello M, Lucchinetti CF, Weinshenker BG, Pittock SJ, Mandrekar J, Thapa P, McKeon A. Azathioprine: tolerability, efficacy, and predictors of benefit in neuromyelitis optica. Neurology 2011;77:659-66.

22. Qiu W, Kermode AG, Li R, Dai Y, Wang Y, Wang J, Zhong X, Li C, Lu Z, Hu X. Azathioprine plus corticosteroid treatment in Chinese patients with neuromyelitis optica. J Cli Neurosci 2015;22:1178-82.

23. Huh SY, Kim SH, Hyun JW, Joung AR, Park MS, Kim BJ, Kim HJ. Mycophenolate mofetil in the treatment of neuromyelitis optica spectrum disorder. JAMA Neuro 2014;171:1372-8.

24. Weinstock-Guttman B, Ramanathan M, Lincoff N, Napoli SQ, Sharma J, Feichter J, Bakshi R. Study of mitoxantrone for the treatment of recurrent neuromyelitis optica (Devic disease). Arch Neurol 2003;63:957-63.
25. Kim SH, Kim W, Park MS, Sohn EH, Li XF, Kim HJ. Efficacy and safety of mitoxantrone in patients with highly relapsing neuromyelitis optica. Arch Neurol 2011;68:473-9.

26. Kitley J, Elsone L, George J, Waters P, Woodhall M, Vincent A, Jacob A, Leite MI, Palace J. Methotrexate is an alternative to azathioprine in neuromyelitis optica spectrum disorders with aquaporin-4 antibodies. J Neurol Neurosurg Psychiatry 2013; 84:918-21.

27. Watanabe S, Misu T, Miyazawa I, Nakashima I, Shiga Y, Fujihara K, Itoyama Y. Low-dose corticosteroids reduce relapses in neuromyelitis optica: a retrospective analysis. Mult Scler 2007;13:968-74

28. Rossi D, Modena V, Sciascia S, Roccatello D. Rheumatoid arthritis: biological therapy other than anti-TNF. Int Immunopharmacol 2015;27:185-8.

29. Cree BA, Lamb S, Morgan K, Chen A, Waubant E, Genain C. An open label study of the effects of rituximab in neuromyelitis optica. Neurology 2005;64:1270-2.

30. Kim SH, Huh SY, Lee SJ, Joung A, Kim HJ. A 5-year follow-up of rituximab treatment in patients with neuromyelitis optica spectrum disorder. JAMA Neurol 2013;70:1110-7.

31. Yang CS, Yang L, Li T, Zhang DQ, Jin WN, Li MS, Su N, Zhangning N, Liu Q, Shao ZH, Yu C, Shi FD. Responsiveness to reduced dosage of rituximab in Chinese patients with neuromyelitis optica. Neurology 2013;81:710-3.

32. Pittock SJ, Lennon VA, McKeon A, Mandrekar J, Weinshenker BG, Lucchinetti CF, O'Toole O, Wingerchuk DM. Eculizumab in AQP4IgG-positive relapsing neuromyelitis optica spectrum disorders: an open-label pilot study. Lancet Neurol 2013;12:554-62.

33. Phuan PW, Zhang H, Asavapanumas N, Leviten M, Rosenthal A, Tradtrantip L, Verkman AS. C1q-targeted monoclonal antibody prevents complement-dependent cytotoxicity and neuropathology in in vitro and mouse models of neuromyelitis optica. Acta Neuropathol 2013;125:829-40.

34. Levy M, Mealy MA. Purified human C1-esterase inhibitor is safe in acute relapses of neuromyelitis optica. Neurol Neuroimmunol Neuroinflamm 2014;1:e5.

35. Chihara N, Aranami T, Sato W, Miyazaki Y, Miyake S, Okamoto T, Ogawa M, Toda T, Yamamura T. Interleukin 6 signaling promotes anti-aquaporin 4 autoantibody production from plasmablasts in neuromyelitis optica. Proc Natl Acad Sci U S A 2011;108:3701-6.

36. Uzawa A, Mori M, Sawai S, Masuda S, Muto M, Uchida T, Ito S, Nomura F, Kuwabara S. Cerebrospinal fluid interleukin-6 and glial fibrillary acidic protein levels are increased during initial neuromyelitis optica attacks. Clin Chim Acta 2013 5;421:181-3.

37. Uzawa A, Mori M, Ito M, Uchida T, Hayakawa S, Masuda S, Kuwabara S. Markedly increased CSF interleukin-6 levels in neuromyelitis optica, but not in multiple sclerosis. $J$ Neurol 2009;256:2082-4

38. Araki M, Aranami T, Matsuoka T, Nakamura M, Miyake S, Yamamura T. Clinical improvement in a patient with neuromyelitis optica following therapy with the anti-IL-6 receptor monoclonal antibody tocilizumab. Mod Rheumatol 2013;23:827-31.

39. Kieseier BC, Stuve O, Dehmel T, Goebels N, Leussink VI, Mausberg AK, Ringelstein M, Turowski B, Aktas O, Antoch G, Hartung HP. Disease amelioration with tocilizumab in a treatment-resistant patient with neuromyelitis optica: implication for cellular immune responses. JAMA Neurol 2013;70:390-3.

40. Ayzenberg I, Kleiter I, Schroder A, Hellwig K, Chan A, Yamamura T, Gold R. Interleukin 6 receptor blockade in patients with neuromyelitis optica nonresponsive to anti-CD20 therapy. JAMA Neurol 2013;70:394-7.

41. Jarius S, Paul F, Franciotta D, Ruprecht K, Ringelstein M, Bergamaschi R, Rommer P, Kleiter I, Stich O, Reuss R, Rauer S, Zettl UK, Wandinger KP, Melms A, Aktas O, Kristoferitsch W, Wildemann B. Cerebrospinal fluid findings in aquaporin-4 antibody positive neuromyelitis optica: results from 211 lumbar punctures. $J$ Neurol Sci 2011;306:82-90.

42. Shimizu F, Sano Y, Takahashi T, Haruki H, Saito K, Koga M, Kanda T. Sera from neuromyelitis optica patients disrupt the blood-brain barrier. J Neurol Neurosurg Psychiatry 2012;83:288-97.

43. Vincent T, Saikali P, Cayrol R, Roth AD, Bar-Or A, Prat A, Antel JP. Functional consequences of neuromyelitis optica-IgG astrocyte interactions on blood-brain barrier permeability and granulocyte recruitment. J immunol 2008;181:5730-7.

44. Tasaki A, Shimizu F, Sano Y, Fujisawa M, Takahashi T, Haruki H, Abe M, Koga M, Kanda T. Autocrine MMP-2/9 secretion increases the BBB permeability in neuromyelitis optica. $J$ Neurol Neurosurg 
Psychiatry 2014;85:419-30.

45. Mealy MA, Shin K, John G, Levy M. Bevacizumab is safe in acute relapses of neuromyelitis optica. Clin Exp Neuroimmunol 2015;6:413-8

46. Lucchinetti CF, Mandler RN, McGavern D, Bruck W, Gleich G, Ransohoff RM, Trebst C, Weinshenker B, Wingerchuk D, Parisi JE, Lassmann H. A role for humoral mechanisms in the pathogenesis of
Devic's neuromyelitis optica. Brain 2002;125:1450-61.

47. Saadoun S, Waters P, MacDonald C, Bell BA, Vincent A, Verkman AS, Papadopoulos MC. Neutrophil protease inhibition reduces neuromyelitis optica-immunoglobulin G-induced damage in mouse brain. Ann Neurol 2012;71:323-33.

48. Zhang H, Verkman AS. Eosinophil pathogenicity mechanisms and therapeutics in neuromyelitis optica. J Clin Invest 2013;123:2306-16. 